\title{
Short Term Load Forecasting Research Based on Electricity Big Data
}

\author{
Hu Wei ${ }^{1}$, Ma Qian ${ }^{1}$, Fang Chao ${ }^{2}$, Xiong Zheng $^{2}$,Ji Cong ${ }^{2, a}$ and Zhong Chunlin ${ }^{2}$ \\ 1 Jiangsu Electric Power Company, Nanjing210024, China; \\ 2Jiangsu Frontier Electric Technology CO.,LTD, Nanjing 211102 China \\ ajcxx01@163.com
}

\begin{abstract}
Keywords: Electricity Information Acquisition System; Electricity Big Data; Short-term Load Forecasting
\end{abstract}

\begin{abstract}
With global information technology development, the era of big data is in the offing. Power industry is closely related with people's livelihood and it is necessary to introduce big data technology to improve its economy and reliability. The completion of Jiangsu Electricity Information Acquisition System and the accumulation of historical information both laid a rich foundation for the study of the electricity big data. Jiangsu Electricity Power Company has an advantage and it is necessary to take full advantage of electricity big data to carry out related researches. Based on electricity big data, research works on short-term load forecasting were carried out in this paper, which had achieved some results and made a certain contribution for the promotion of big data technology in Jiangsu Electricity Power Company.
\end{abstract}

\section{Introduction}

With global information technology development, data growth explosion occurred, followed by the era of big data. Foreign countries carried out big data researches at first moment, and most researchers are from Internet companies which are pioneers of information technology research and application. IBM as a pioneer of Big Data research, has carried out a lot of research works and achieved certain results and applications in power grid and power generation[1-2]. Domestic electricity big data research also followed the pace of International researches and carried out related works [3]. Experts in power systems also carried out a series of research works, such as build knowledge model from big data [4], residential electricity behavioral analysis [5], and load forecasting based on big data[6].

Jiangsu Electric Power Company comprehensively completed electricity consumption acquisition system which contains more than 38 million users' real-time power consumption information in 2014, thus we got a collection of 38 million users' electricity consumption data. We formed a power consumption big data center contained more than 10 years' electricity, industry expansion, weather, product consumption and other historical data. Based on power consumption big data center, combining with the application of big data technology and a variety of advanced algorithms, Jiangsu Electric Power Company did some researches:

(1) Comprehensively analyzed the behavior of electricity load characteristics of more than 38 million users;

(2) Established the relationship between electricity and temperature, humidity, holidays and other factors, constructed over all 300,000 users and 450,000 transformer model;

(3) Launched research for the short-term load forecasting.

\section{Load impact model based on big data}

In the rapid development of power system information data, we should make full use of existing electricity information big data resources, analyze the relationship between industry and temperature, humidity, thereby improving the accuracy of power system load modeling and load forecasting, and ultimately provide some data support for the electricity power dispatching. 
To quantify the extent of the temperature on the load, improve the accuracy of customer load meteorological model and eliminate the influence of factors of the natural growth rate, holidays, this paper takes three or more years of historical data to build load meteorological models. Construction of concrete steps is shown below. Since the workday and weekend user load show entirely different habits, so the calculation of user load impact factor is shown below.

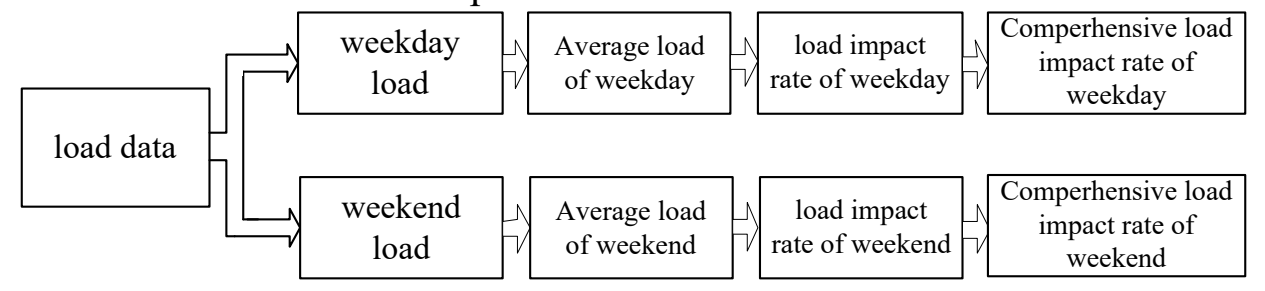

Fig.2 Load meteorological modeling process

Take workday for example, the modeling process is as follows:

1)Extracting load data of workday in the last $Y$ years and calculating the average load of 96 points per year in recently $\mathrm{Y}$ years.

$$
\bar{P}_{(y, i)}=\sum_{k \in W} P_{(y, d, i)} / D_{y}
$$

Where: the subscript $y$ as the year, $d$ as the date, $i$ as the order of 96-point load; $\bar{P}_{(y, i)}$ for the users' average load in ith point of workday in year $y ; P_{(y, d, i)}$ for users' ith load data of the $d$ th day in year $y$; $D_{y}$ for number of workday in year $y ; W$ for the collection of workday in year $y ; k \in W$ means $k$ belongs to set $W$.

$$
R_{(y, d, i)}=\left(P_{(y, d, i)}-\bar{P}_{(y, i)}\right) / \bar{P}_{(y, i)}
$$

Where: $R_{(y, d, i)}$ for user's ith load impact rate of the $d$ th workday in year $y$.

According to temperature shift, the recent $\mathrm{Y}$ years' 96 point load impact rate for all weekday is classified to the corresponding temperature position. Since the temperature has been divided into $>$ higher than 40 , lower than -4 ,and $-4 \sim 40$, and it will form a $45 \times 96$ temperatures - load impact rate sequence.

2)Calculating load impact rate point by point.

$$
R_{(y, d, i, t)} \in S_{(i, t)}
$$

Where: $t$ is temperature. Formula (3) means taking impact rate where time $=i$, temperature $=t$ into the same set.

3)Calculating comprehensive temperature-load impact rate.

In order to reflect the effect of temperature impact on load, calculating average load impact rate under each temperature $S_{(i, t)}$ stalls.

After the above computing steps, users load impact model has been built, three-dimensional model is shown in Figure 3. 


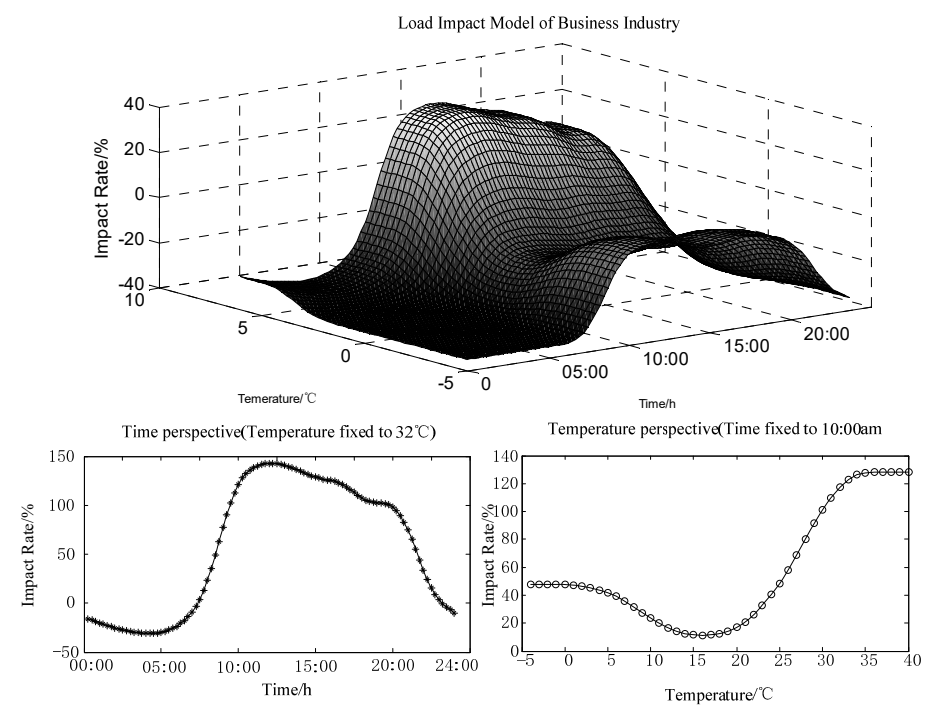

Fig. 3 Load impact model of business industry

The construction of electricity-meteorological influence model laid theoretical basis and data support for the analysis of diversified customer electricity behavior and improve the accuracy of load forecasting.

\section{Short-term load forecasting based on electricity big data}

Short-term load forecasting is influenced by weather conditions and load characteristics. This paper takes similar day as a reference for the daily load forecasting, and considers the region and industry load characteristics, combining with weather forecast information and finally proposing a short-term load forecasting method which is based on the industry area subdivision. The process is as follows.

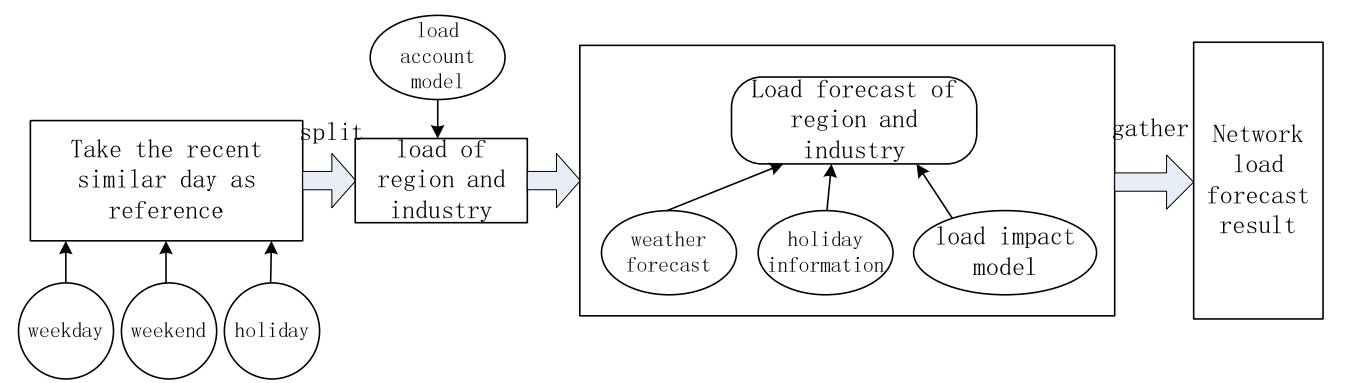

Fig.4 Short-term load forecasting process based on the electricity big data

(1)Selecting similar days. Selecting load of recent similar days as the original load. For example, taking last weekday as weekday similar days, taking last weekend as weekend similar days and taking holiday of last year as holiday similar days.

(2)Splitting the load by region and industry. According to the proportion of total load for regions load and industry load, the paper establishes accounting model and splits similar days load into regional load and industry load based on this model.

(3)Forecasting the load by region and industry. Based on regional and industry load meteorological model, and considering the weather forecast and holiday information, the paper can forecast the load by region and industry.

(4)Forecasting short-term load of Jiangsu Province. Considering the statistical error between the province's network load and the load of region and industry, and combining with the historical trend of regional and industry load, the paper calculates the comprehensive impact rate $t$ of region load and industry load, and finally achieves the province's load forecast results by the proportion information of load impact rate.

Taking Jiangsu network load forecasting for example, and verifing the proposed big data algorithm for load forecasting. 


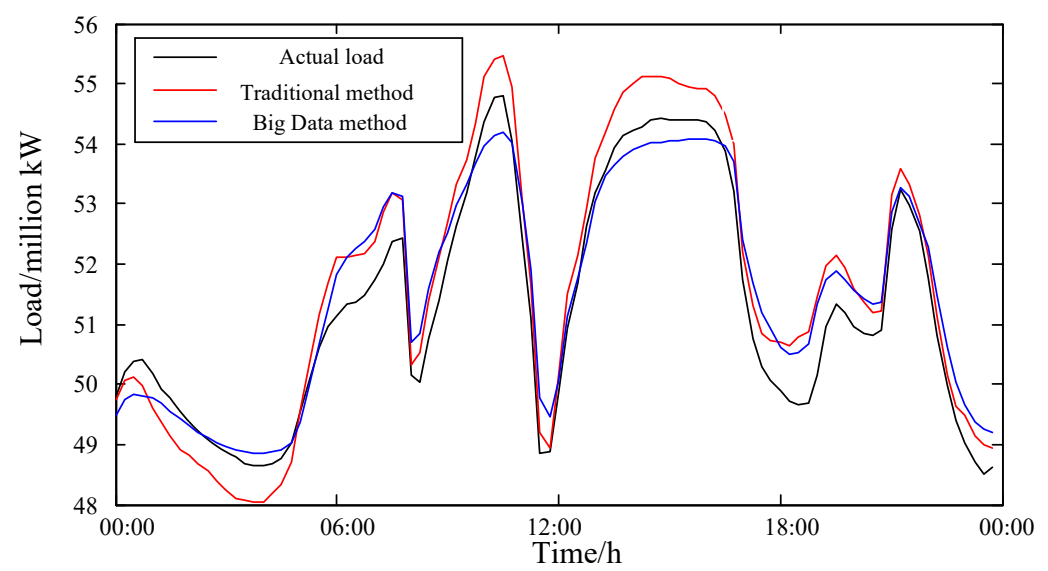

Fig. 5 Short-term load forecasting result

The green line is actual load curve, yellow line is forecasting load curve by traditional load forecasting methods and the blue line is obtained by big data method. The graph shows the result of big data method is better than that of traditional method, which is mainly due to the proposed method which accounts the temperature difference between cities, electricity characteristics of different industries into consideration and thus can improve the accuracy of load forecasting.

\section{Summary}

This paper carried out the following researches based on electricity big data:

(1)Based on the current electricity big data resources, the relationship among power, electricity and temperature, humidity was analyzed.

(2)Takeing region, industry power characteristics and weather forecast information into consideration, puting forward short-term power load forecasting method based on region and industry. Test result shows the effectiveness of the method in this paper.

This paper carries out some researches in electricity big data, but it is just a start of electricity big data application, and there are more researches and challenges to be conducted.

\section{References}

[1] IBM. Power wind site down time IBM's solution in power industry. http://www31.ibm.com/solutions/cn/industries/energy/thankyou/energy_wp.shtml.

[2] IBM . IBM smart grid solution (power grid). http://www.ibm.com/smarterplanet/cn/zh/ smart_grid/ ideas/index.html.

[3] Zhang Pei, Yang Huafei, Xu Yuanbin, et al. Electricity big data and Its Application Scenarios in Power Grid. Proceedings of the CSEE, 2014, vol.34, pp: 85-92.

[4] Huang Yanhao, Zhou Xiaoxin. Knowledge model for electric electricity big data based on ontology and semantic web. CSEE Journal of Power and Energy Systems, 2015, vol.35, pp.19-27.

[5] Zhang Suxiang, Liu Jianming, Zhao Bingzhen, et al. Cloud computing-based analysis on residential electricity consumption behavior. Power System Technology, 2013, vol.37, pp.1542-1546.

[6] Zhang Suxiang, Zhao Binzhen, Wang Fengyu, et al. Short-term power load forecasting based on big data. Proceedings of the CSEE, 2015, vol.35, pp.37-42. 